\title{
Sharp Boundary Based Transient Electromagnetic Inversion
}

\author{
Rui Guo*, Xin Wu, Liting Rao, Guangyou Fang \\ Key Laboratory of Electromagnetic Radiation and Sensing Technology \\ Chinese Academy of Sciences \\ Beijing, China \\ greencs@163.com
}

\begin{abstract}
It is very important to get a reliable result in transient electromagnetic (TEM) inversion. Most of the traditional inversion algorithms are based on the assumption that resistivity changes smoothly underground, through which we cannot get clear geological interface. With the purpose to represent clear boundary with resistivity discontinuity, this paper applies Minimum Gradient Support (MGS) Functional to TEM inversion. Through the three-layer model test, this method is proved more accurate in determining sharp electrical interface compared with smoothest model inversion. Also, this algorithm does not rely on the starting model and the iterative process is stable.
\end{abstract}

Keywords-transient electromagnetic inversion; sharp boundary; smoothest model inversion; minimum gradient support

\section{INTRODUCTION}

Transient electromagnetic (TEM) method is a kind of electromagnetic detection method of artificial source based on the process of transmitting the primary electromagnetic impulse to underground and analyzing changes of secondary field versus time to get the electrical characters of the medium. Data inversion plays an important role in TEM exploration. Although there are many inversion methods, it is still essential to find more approaches to acquire more accurate results. Predecessors provided lots of methods to improve the reliability and speed of the inversion, such as: OCCAM inversion introduced by Constable ${ }^{[1]}$ and deGroot-Hedlin ${ }^{[2]}$; RRI introduced by Smith and Booker ${ }^{[3]}$; NLCG intorduced by $\operatorname{Rodi}^{[4]}$, and so on. In addition, artificial neural net ${ }^{[5]}$, simulated annealing $^{[6]}$ and multiresolution inversion ${ }^{[7]}$ are also developed. These methods usually use objective function based on the smoothest stable functional, thus the inversion results are too smooth to represent the real geo-electrical interface, which makes the geophysical and geological interpretation difficult.

Another available approach overcoming such problem is to combine smoothest model inversion with Marquardt inversion $^{[8]}$. By assuming the Marquardt staring model according to the smoothest model inversion, the Marquardt inversion can give us a sharp boundary result. However, this method converges unstable.

Portniaguine and Zhdanov ${ }^{[9]}$ introduced Minimum Support (MS) Functional and Minimum Gradient Support (MGS) Functional to minimize the area where strong model parameter variations and discontinuity occur. The method had been applied to real gravity data and successfully tested. Based on the previous research, this paper applies Minimum Gradient Support (MGS) Functional to TEM inversion's objective function. This method helps to generate sharper and more focused curves for geo-electrical structures than using conventional smoothest functionals. Through the three-layer model test, this method is proved more accurate in determining sharp electrical interface compared with smoothest model inversion.

\section{MODEL AND THEORY}

\section{A. Modelling}

The 1-D earth model is applied here. Totally, there are $N$ layers in the model. The thickness of each layer is $h_{i}$, the thickness is $\rho_{i}$, respectively. The radius of the transmitter coil is $a$, and the current is $I$. The secondary field at the center of the receiver coil can be expressed in matrix notation as $A[\boldsymbol{\rho}, \mathbf{h}, I, a]$, where $\boldsymbol{\rho}=\left[\rho_{1}, \rho_{2} . . \rho_{N}\right], \mathbf{h}=\left[h_{1}, h_{2} \ldots h_{N}\right], A[\cdot]$ represents forward modeling functional.

\section{Inversion Method}

Assuming that the thickness of each layer is fixed, and the current and radius of the transmitter coil are known. Let $d_{o b s}$ be some observed data, then the inversion problem can be expressed as

$$
d_{o b s}=A[\boldsymbol{\rho}]
$$

The element $\boldsymbol{\rho}$ is a solution of the inverse problem. However, widely differing numerical geo-electric models may be found in the inversion problem, which may cause almost the same electromagnetic field behavior ${ }^{[10]}$. This lack of uniqueness makes inversion difficult, thus the foundations of the regularization theory were developed by Tikhonov, described $i^{[11]}$, ${ }^{[12]}$ and ${ }^{[13]}$. The objective function can be expressed as

$$
P^{\alpha}(\boldsymbol{\rho})=\phi(\boldsymbol{\rho})+\alpha s(\boldsymbol{\rho})
$$

where the first term on the right is called Misfit Functional, by which we assess the goodness of fit of the model 
predictions to the actual values, and the second the Stabilizing Functional. A stabilizing functional can be used to select the appropriate class of models for inverse problem solution. We call $\alpha$ Regularization Parameter, which describes the tradeoff between the best fitting and most reasonable stabilization [1].

The misfit functional can be written as

$$
\phi(\boldsymbol{\rho})=\left\|W_{d}\left(A[\boldsymbol{\rho}]-d_{o b s}\right)\right\|^{2}
$$

where $W_{d}$ is the data weighting matrix. There are several common choices for a stabilizer. In practice, we select the model that is simplest in some sense, yet still fits the data. One is based on the least square criterion:

$$
s(\boldsymbol{\rho})=\|\boldsymbol{\rho}\|^{2}
$$

Another stabilizer uses a maximum smoothness model:

$$
s(\boldsymbol{\rho})=\|\nabla \boldsymbol{\rho}\|^{2}
$$

It has been successfully used in many inversion schemes (Constable $^{[1]}$, Smith and Booker ${ }^{[3]}$, Zhdanov ${ }^{[14]}$, Zhdanov and Fang $\left.{ }^{[15]}\right)$. In order to minimize the objective function, $s(\boldsymbol{\rho})$ should be small enough. Thus, the change of inversed resistivity fails to describe properly the real blocky geological structures because it is supposed to be as smooth as possible. Using maximum smoothness model stabilizer cannot reflect the sharp geo-electric interface. It can also cause spurious oscillations when $\rho$ is discontinuous ${ }^{[10]}$.

\section{B. Minimum Gradient Support Inversion}

The "smoothness" effect can be diminished by introducing another stabilizer functional which minimizes the area where significant variations of the model parameters and discontinuity occur ${ }^{[9]}$. It is called Minimum Gradient Support Functional.

$$
S_{M G S}(\boldsymbol{\rho})=S_{\beta}(\boldsymbol{\rho})=\int_{V} \frac{\nabla \boldsymbol{\rho} \square \nabla \boldsymbol{\rho}}{\nabla \boldsymbol{\rho} \square \boldsymbol{\rho}+\beta^{2}} d V
$$

$\nabla \boldsymbol{\rho}$ is the gradient of the model, and $\beta$ is a very small number. In the last formula we assume that the function $\nabla \boldsymbol{\rho}$, which describes the model parameters, is given within a onedimensional domain $V . S_{M G S}(\boldsymbol{\rho})$ can be written as another form:

$$
S_{M G S}(\boldsymbol{\rho})=\operatorname{spt} \nabla \boldsymbol{\rho}-\beta^{2} \int_{s p t \nabla \boldsymbol{\rho}} \frac{1}{\nabla \boldsymbol{\rho} \nabla \nabla \boldsymbol{\rho}+\beta^{2}} d V
$$

where $\operatorname{spt} \nabla \boldsymbol{\rho}$ is the combined closed subdomains of $V$ where $\nabla \boldsymbol{\rho} \neq 0$. We can see that $s_{\beta}(\boldsymbol{\rho}) \rightarrow \operatorname{spt} \nabla \boldsymbol{\rho}$ if $\beta \rightarrow 0$. Thus, the functional $s_{M G S}(\boldsymbol{\rho})$ can be treated as a functional proportional to the gradient support when $\beta \rightarrow 0$. A sharp and focused image of the inversed model can be generated by using MGS functional.
At last, we get the objective function of the inverse problem:

$$
P^{\alpha}(\boldsymbol{\rho})=\left\|W_{d}\left(A[\boldsymbol{\rho}]-d_{o b s}\right)\right\|^{2}+\alpha S_{M G S}(\boldsymbol{\rho})
$$

This unconstrained functional is minimum when $\nabla_{\rho} P^{\alpha}$, the gradient of $P^{\alpha}$ with respect to $\boldsymbol{\rho}$, vanishes. After a little algebra, we get

$$
\begin{gathered}
\boldsymbol{\rho}_{i}=\left[\left(W_{d} \mathbf{J}\right)^{T}\left(W_{d} \mathbf{J}\right)+\alpha\left(W_{\beta i} \nabla\right)^{T}\left(W_{\beta i} \nabla\right)\right]^{-1} W_{i} \hat{d}_{i} \\
\hat{d}_{i}=d_{o b s}+\mathbf{J} \boldsymbol{\rho}_{i-1}-A\left[\boldsymbol{\rho}_{i-1}\right] \\
W_{\beta}=\frac{1}{\sqrt{\nabla \boldsymbol{\rho} \square \boldsymbol{\rho}+\beta^{2}}}
\end{gathered}
$$

when $\nabla_{\rho} P^{\alpha}=0 . \mathbf{J}$ is called Jacobian or gradient matrix:

$$
\mathbf{J}=\nabla_{\rho} A[\boldsymbol{\rho}]
$$

Once a starting model is defined, the equations above can be calculated by iterative scheme to get the final inversion results: each preceding vector is used as the starting approximation for the next. When the misfit is smaller than the desired misfit, the iteration ends. The result will be the model of focused curves with the specified misfit.

\section{EXAMPLES}

There are two examples of the application of our inversion technique presented here. Our model is a three-lay earth model. The thickness from top to bottom of each layer is $100 \mathrm{~m}, 150 \mathrm{~m}$, half space, and the resistivity is $300 \Omega \cdot m, 100 \Omega \cdot m, 300 \Omega \cdot m$. The radius of the transmitter coil is $100 m$, and the receiver, which measures the vertical magnetic field, is placed at the center of the transmitter coil. The current is set to be unity.

\section{A. Theoretical Data Inversion}

The theoretical data is calculated from forward modeling. Fig. 1 shows the results of MGS inversion and smoothest model inversion. The MGS inversion curve is more focused and has a larger range of gradient than smoothest model inversion. By using MGS method, the inversed resistivity can reach $100 \Omega \cdot m$ from $135 \mathrm{~m}$ to $227 \mathrm{~m}$ underground, while the smoothest model inversion can only reach $109 \Omega \cdot m$ at $191 \mathrm{~m}$ underground, which illustrates that MGS method can reflect the geo-electrical interface more clearly than smoothest model inversion. Fig. 2 shows that forward modeling results (blue squares) calculated by inversed model match well with the red line, which represents the theoretical forward modeling.

\section{B. Inversion with Noise}

The noise is added to the theory data with the peak-to-peak value of $0.01 \mathrm{nV} / \mathrm{m}^{2} / \mathrm{A}$. The late time data is omitted because of its low SNR. Only 28 data points are used here. 


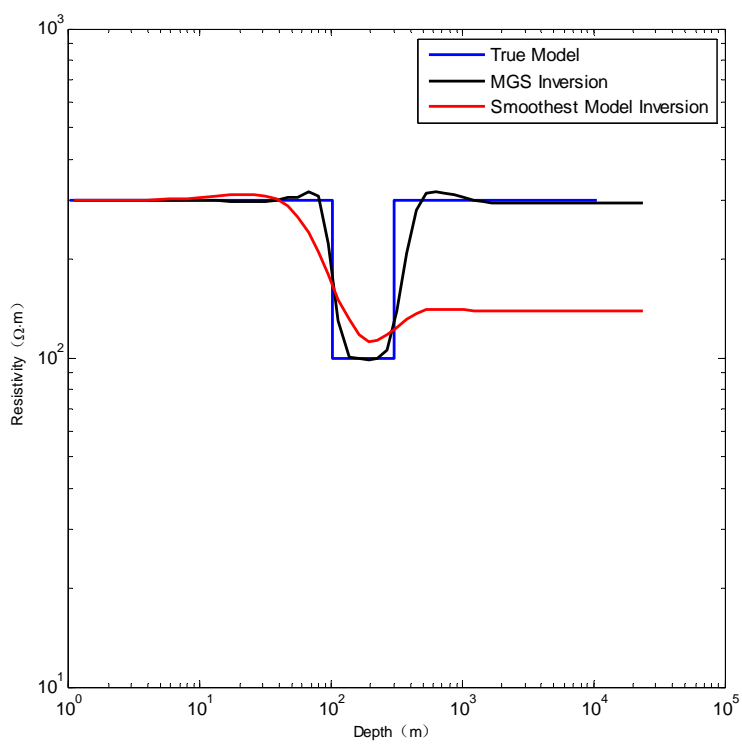

Fig. 1. Inversed results of MGS and smoothest model. The blue line represents true model, and the black line represents the MGS inversion result, and the red line represents smoothest model inversion result.

Fig. 3 shows the inversion curves of two methods. The smoothest model inversion cannot reflect the true earth structure because of the data abundance. However, MGS method shows us a more focused curve due to the fact that it minimizes the area where variations occur.

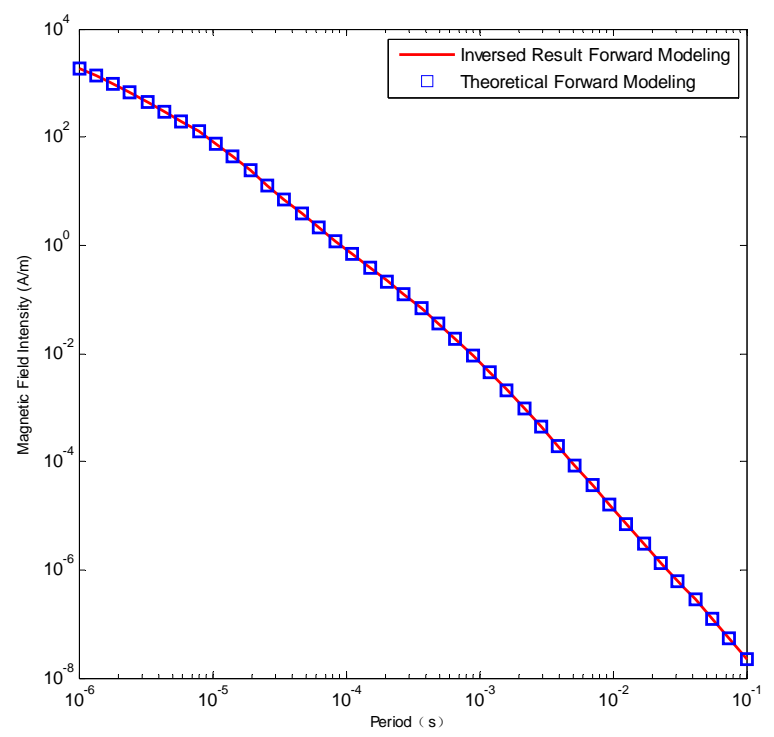

Fig. 2. The forward modeling curves of true three-layer structure and MGS inversion results. The red line is from true structure forward modeling, and the blue squares represent MGS inversion results.

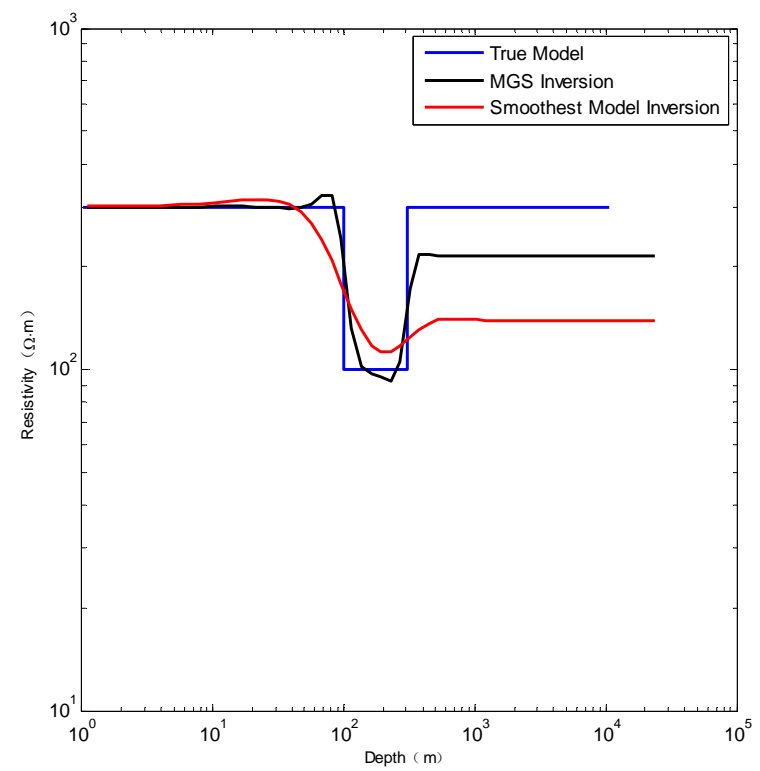

Fig. 3. Inversed results of MGS and smoothest model with noise. The peakto-peak value of the noise is $0.01 \mathrm{nV} / \mathrm{m}^{2} / \mathrm{A}$. The blue line represents true model, and the black line represents the MGS inversion result, and the red line represents smoothest model inversion result.

Fig. 4. shows that although there are only 28 data points used in the inversion, the forward modeling results (blue squares) calculated by inversed model match well with the red line, which is the theory forward modeling.

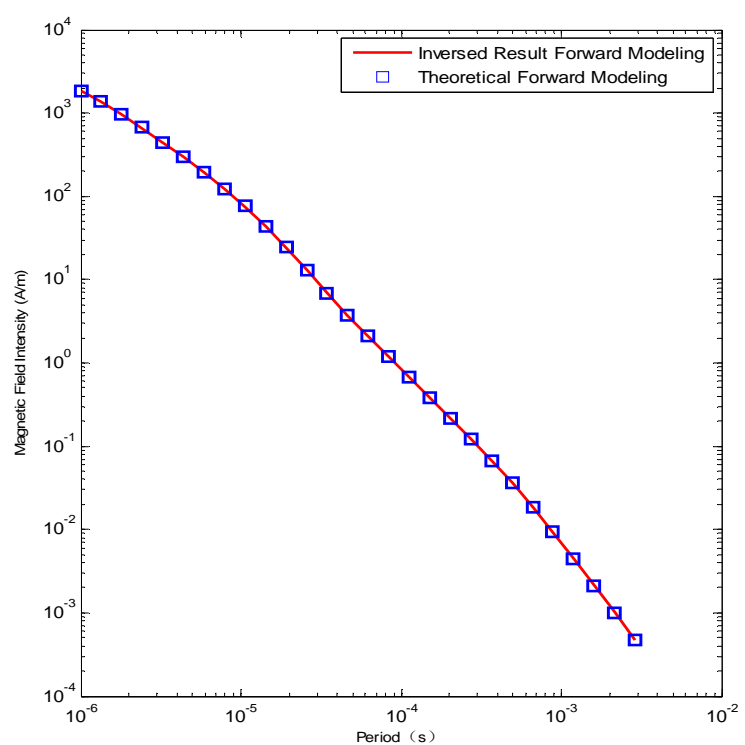

Fig. 4. The forward modeling curves of true three-layer structure and MGS inversion results. The red line is from true structure forward modeling, and the blue squares represent MGS inversion results. Only 28 data points are used to inverse. 


\section{CONCLUSIONS}

Even though that the traditional maximum smoothest model inversion has been widely used in TEM exploration, it cannot show us the real blocky geological and geo-electrical structures. This paper introduces another method to achieve the TEM data inversion by applying the MGS Functional to the objective functions. By using the MGS stabilizer, the "smoothness" effect can be diminished and the area with variations of parameters can be focused. In addition, this method does not rely on the starting model and converges stable. A three-layer earth model is established to test this method. The result shows that compared with the smoothest model inversion, MGS inversion presents us the inversed parameters with a sharper boundary, and makes the geological and geo-electrical interface and the blocky body be shown clearer and more accurate. Thus, the problem that the oblique geological and geo-electrical interface cannot be distinguished can be solved.

\section{ACKNOWLEDGMENT}

This study is supported by R\&D of Key Instruments and Technologies for Deep Resources Prospecting (the National R\&D Projects for Key Scientific Instruments), Grant No.ZDYZ2012-1-03-05 ATEM flight test, data process and interpretation software technology.

\section{REFERENCES}

[1] Constable S C and Parker R L. "Occam's inversion: A practical algorithm for generating smooth models from electromagnetic sounding data”. Geophysics . vol. 52, 1987,pp. 289-300.

[2] deGroot-Hedlin C and Constable S, “Occam’s inversion to generate smooth, two-dimensional models from magnetotelluric data”, Geophysics ,vol. 55,1892, pp. 1613-1624.

[3] Smith J T and Booker J R, "Rapid inversion of two- and threedimensional magnetotelluric data” ,Geophys. Res, 1991, pp. 39053922

[4] Rodi W, and Machie R L, "Nonlinear conjugate gradients algorithm for 2-D magnetotelluric inversion”, Geophysics, vol. 66, 2001,pp.174-187

[5] Wang J Y, "Review of electrical prospecting for petroleum in China”, Progress in Exploration Geophysics, vol. 29, 2006, pp.77-82

[6] Yang $\mathrm{H}$, Wang $\mathrm{J} \mathrm{L}$ and $\mathrm{Wu} \mathrm{J} \mathrm{S}$, "Constrained joint inversion of magnetotelluric and seismic data using simulated annealing algorithm”, Chinese J. Geophys, vol. 45 , 2002, pp. 723-734

[7] Xu Y X, and Wang J Y, "A multiresolution inversion of onedimensional magnetotelluric data”, Chinese J. Geophys, vol. 41 , 1998,pp. 704-711

[8] Marquardt D W, “An algorithm for least- squares estimation of nonlinear parameters”, J. Soc. Ind. Appl. Math.,vol. 11, 1963, pp.431-441

[9] Portniaguine O. and Zhadanov M. S, "Focusing geophysical inversion images”,Geophysics, vol. 64,1999, pp. 874-887

[10] Michael S. Zhdanov, "Geophysical electromagnetic theory and methods”,Elsevier Publications,2009

[11] Tikhonov, A. N., "Solution of ill-posed problems and the regularization method”, Soviet Math Dokladi, vol. 4, 1963, pp.1035-1038.

[12] Tikhonov, A. N., "Regularization of ill-posed problems”, Soviet Math Dokladi,vol.4 , 1963, pp. 1624-1627.

[13] Tikhonov, A. N., "Mathematical geophysics", Moscow State University, 1999,

[14] Zhdanov M. S., “Tutorial: regularization in inversion theory”, Colorado School of Mines, 1993,

[15] Zhdanov M. S. and S. Fang, "3-D quasi-linear electromagnetic inversion”, Radio Science, vol. 31, 1996, pp. 741-754 